\title{
Resonant multiphoton processes in the soft-x-ray regime
}

\author{
M. Martins, ${ }^{1, *}$ M. Wellhöfer, ${ }^{1}$ A. A. Sorokin, ${ }^{2,3}$ M. Richter, ${ }^{2}$ K. Tiedtke, ${ }^{4}$ and W. Wurth ${ }^{1}$ \\ ${ }^{1}$ Institut für Experimentalphysik, Universität Hamburg, Luruper Chaussee 149, D-22761 Hamburg, Germany \\ ${ }^{2}$ Physikalisch-Technische Bundesanstalt, Abbestraße 2-12, D-10587 Berlin, Germany \\ ${ }^{3}$ Ioffe Physico-Technical Institute, Polytekhnicheskaya 26, 194021 St. Petersburg, Russia \\ ${ }^{4}$ Deutsches Elektron-Synchrotron, Notkestraße 85, D-22603 Hamburg, Germany \\ (Received 30 October 2008; revised manuscript received 17 April 2009; published 17 August 2009)
}

\begin{abstract}
The multiphoton ionization of neon atoms was studied in the focus of the soft-x-ray free-electron laser FLASH by photoion spectroscopy. The photon energy was chosen in the range of $\mathrm{Ne}^{+} 2 p \rightarrow n \ell$ resonances between 41 and $42 \mathrm{eV}$. The experiments were performed at the PG0 branch of the FLASH monochromator beamline while measuring the spectral distribution of every single FLASH pulse. By correlating the ratio of doubly and singly charged neon ions generated and the measured photon energy distribution, the enhancement of this ratio due to resonant $\mathrm{Ne}^{+}$excitation was found.
\end{abstract}

DOI: 10.1103/PhysRevA.80.023411

PACS number(s): $32.80 . \mathrm{Fb}, 32.80 . \mathrm{Hd}$

\section{INTRODUCTION}

With the advent of new powerful soft-x-ray sources based on free-electron laser (FEL) [1,2] and high-harmonic generation techniques [3], multiphoton processes can be studied for the first time also at photon energies above ionization thresholds. In contrast to conventional laser sources for optical radiation, the new soft-x-ray lasers can also access innervalence and core-level electrons. Thus, all advantages of core level spectroscopy, e.g., its site and element specificity, can now be combined with the potentials of multiphoton processes, e.g., the study of fast dynamical processes by pumpprobe spectroscopy. An outstanding feature in core-level spectroscopy are resonant inner-shell excitations, because they can increase the core-level photoionization cross sections quite dramatically. Furthermore, they open a way to obtain a chemical contrast or to excite, in a complicated multiatom system, a well-defined site.

The importance of inner-shell contributions and resonant core-level excitations to multiphoton processes is still an open question [4,5]. Sorokin et al. [5] showed that, in the case of the $\mathrm{Xe} 4 d-\epsilon f$ continuum resonance, more than 50 photons may be absorbed within 10-20 fs resulting in highly charged Xe ions. Current theoretical models do not give a satisfying description for these experimental findings [6,7]. However, two recent theoretical studies on the two-photon ionization of neon by Hamonou et al. [8,9], using the $R$-matrix approach, have pointed out the importance of innershell electron correlation and emission processes on multiphoton ionization in the soft-x-ray regime. The two-photon ionization cross section, e.g., depends strongly on the chosen configuration interaction basis for the target states or the position of two-photon resonances [8].

In order to perform resonant core-level spectroscopy and hit a resonant state, a well-defined photon energy is mandatory. However, the photon energy distribution of FEL radiation based on self-amplified spontaneous emission, i.e., a statistical process, is strongly varying from shot to shot. At

\footnotetext{
*michael.martins@desy.de
}

FLASH, this disadvantage can be overcome, e.g., by using the FLASH plane grating monochromator. This would, in fact, strongly reduce the available photon density and the probability of multiphoton and nonlinear processes. Hence, we have chosen another possibility in the present study: it is the measurement of the spectral distribution for each individual FEL pulse and the correlation of the acquired photoionization data with these spectra.

\section{EXPERIMENTS}

To realize our experiment, we have used the so-called PG0 beamline of the Free-electron LASer in Hamburg FLASH. As demonstrated in Fig. 1, it represents a special branch of the FLASH plane grating monochromator beamline [10]. At the PG0 beamline, it is possible to use the zero-order light from the grating to perform an experiment and to acquire simultaneously the spectral information for each single FLASH pulse in the first order at the so-called PG2 branch.

For our experiments, FLASH was operating in the single bunch mode with a mean photon energy in the range from 41.4 to $41.5 \mathrm{eV}$, i.e., just above the $\mathrm{Ne}^{+} \rightarrow \mathrm{Ne}^{2+}$ ionization threshold at $41.0 \mathrm{eV}$ [11]. The FEL spectra were recorded on a single-shot basis by using a Ce-doped YAG crystal to convert the soft $\mathrm{x}$ rays into visible light and an Andor Intensified CCD (ICCD) camera system, mounted in the dispersion



FIG. 1. (Color online) Experimental setup at the PG0 beamline. The photoionization experiment using an ion TOF spectrometer is performed by taking the undispersed light in the zero order of the grating, whereas the first-order light is recorded by a Ce:YAG crystal and an ICCD camera at the position of the exit slit of the PG2 branch to monitor the spectral distribution of the FEL pulses. 




FIG. 2. (Color online) $\mathrm{Ne}^{2+}-$ to- $-\mathrm{Ne}^{+}$ratio for different photon exposures. The solid lines represent model curves which correspond to $\mathrm{Ne}^{+} \rightarrow \mathrm{Ne}^{2+}$ cross-section values of $\sigma=3.1$ (red), 1 , and $7 \mathrm{Mb}$ (blue).

plane of the PG2 monochromator beamline. The photoionization experiments were performed in the zero-order PG0 branch using an ion time-of-flight (TOF) mass spectrometer described elsewhere [12]. Within the PG0 branch, the undispersed FEL beam was focused by a toroidal mirror with a focal distance of $0.5 \mathrm{~m}$ down to a focal spot size of about $40 \times 58 \mu \mathrm{m}^{2}$ [13]. The spot area was measured using the method described in Ref. [12], whereas the shape of the spot was taken from ray-trace calculations.

We accumulated the data of 3000 single FLASH pulses and retrieved the number of photons from the FLASH gasmonitor detectors [14], the mean photon energy, and the rms of the photon energy from the ICCD spectra, and the strength of the $\mathrm{Ne}^{+}$and $\mathrm{Ne}^{2+}$ signals using the ion TOF. The neon pressure in the ion TOF chamber was about $10^{-6} \mathrm{hPa}$. The TOF data were taken by means of a fast digitizer card (Acqiris DP282) by digitizing directly the signals from an open electron multiplier. All data were sent to the FLASH data acquisition system and stored in ROOT files.

\section{RESULTS AND DISCUSSION}

In Fig. 2, the ratio of $\mathrm{Ne}^{2+}$ to $\mathrm{Ne}^{+}$is depicted as a function of the photon exposure. As shown by Sorokin et al. [15], for moderate photon exposure levels below 5 $\times 10^{16}$ photons $/ \mathrm{cm}^{2}$, the ratio of the $\mathrm{Ne}^{2+}$-to- $\mathrm{Ne}^{+}$signals shows a linear dependence, explained by the photoionization sequence $\mathrm{Ne} \rightarrow \mathrm{Ne}^{+} \rightarrow \mathrm{Ne}^{2+}$. From the slope of this ratio, the absolute cross section for the second step, i.e., the $\mathrm{Ne}^{+}$ $\rightarrow \mathrm{Ne}^{2+}$ ionization, can be derived by data fitting. In our case the fit value amounts to $3.1 \pm 1.1 \mathrm{Mb}$. This is comparable to the result of Covington et al. [11] obtained at the Advanced Light Source for the photoionization of a $\mathrm{Ne}^{+}$ion beam at the photon energy of $41.2 \mathrm{eV}$, where a cross section of $4.45 \mathrm{Mb}$ was measured.

At the photon exposure levels we have applied for our experiments, the $\mathrm{Ne}^{2+}$ signal was rather low and the signalto-noise ratio comparably high. However, the scatter of the single pulse measurements around the fit curve in Fig. 2 is much larger than expected. This large scatter in the $\mathrm{Ne}^{2+}$-to- $\mathrm{Ne}^{+}$ratio can be explained by the scatter in the excitation energies. Simultaneous measurement of the photon

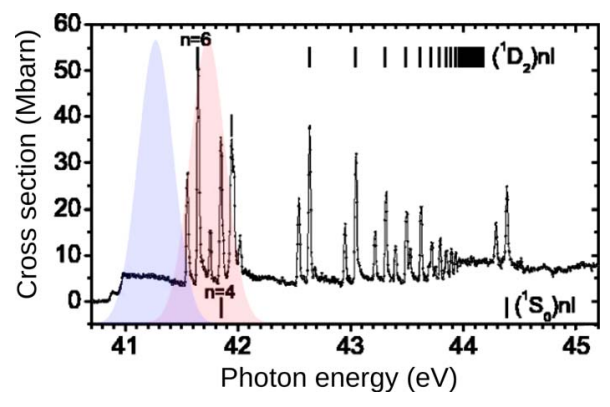

FIG. 3. (Color online) $\mathrm{Ne}^{+}$spectrum taken from Ref. [11]. The shaded Gaussian profiles show two typical FLASH pulses with different mean photon energies. The energy positions correspond to the nonresonant and the resonant areas in Fig. 4.

energies shows that these energies cover a range just above the $\mathrm{Ne}^{+}$threshold where a series of Rydberg excitations into $\mathrm{Ne}^{+} 2 s^{2} 2 p^{4}\left({ }^{1} D_{2}\right) n \ell$ states was observed by Covington et al. [11]. From Fig. 3 one can see that $\mathrm{Ne}^{+}$created by a first photon may be further ionized in a sequential process via resonance states if the photon energies are above $41.5 \mathrm{eV}$ leading, finally, to $\mathrm{Ne}^{2+}$. In Fig. 3, the data of Covington et $a l$ are shown together with two typical FLASH pulses. The photon energy jitter of FLASH and the rms energy width are on the order of $1 \%$, respectively. Hence, an excitation in the energy range from 41.2 to $41.7 \mathrm{eV}$ might result in an excitation just below the resonances (low energy FEL peak) or a strongly resonant excitation (high energy FEL peak), with strong consequences for the creation of $\mathrm{Ne}^{2+}$.

To prove this idea, we have correlated the $\mathrm{Ne}^{2+}-$ to- $-\mathrm{Ne}^{+}$ ratio with the photon energy measured at the PG2 branch. In Fig. 4, the photon-energy-dependent ratio is depicted for a constant photon exposure of $3 \times 10^{16}$ (photons $/ \mathrm{cm}^{2}$ )/pulse (low energy area in Fig. 2). The photon energy is given by the upper edge of the photon energy spectrum of each individual pulse, which is defined as the mean photon energy plus the rms energy width of the photon pulses. The data were binned with respect to the photon energy and the error bars for the photon energy are given by the width of these bins.

Figure 4 obviously confirms our idea that the strong scatter in Fig. 2 is due to the photon energy jitter of FLASH and

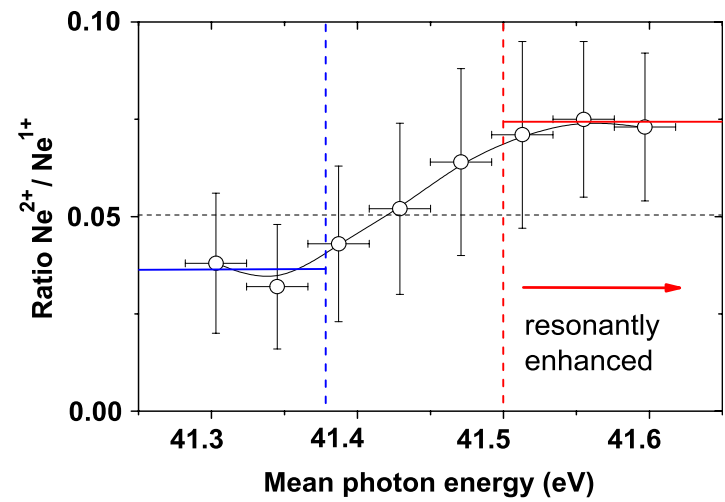

FIG. 4. (Color online) $\mathrm{Ne}^{2+}-$ to- $-\mathrm{Ne}^{+}$ratio as a function of the mean exciting photon energy of FLASH for a photon exposure of $3 \times 10^{16}$ photons $/ \mathrm{cm}^{2}$. For photon energies above $41.4 \mathrm{eV}$, an enhancement by a factor of 2 is found. 


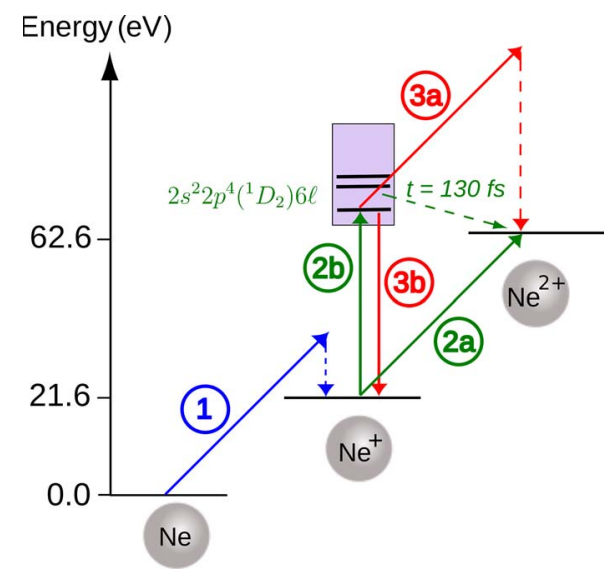

FIG. 5. (Color online) Excitation scheme for resonant multiphoton processes in Ne. A first FLASH pulse will ionize $\mathrm{Ne}$ atoms to $\mathrm{Ne}^{+}(1)$. Further photons will excite resonantly a further $2 p$ electron into $6 \ell$ states $(2 b)$ with a typical lifetime of $130 \mathrm{fs}$. Within the FEL pulse duration of 20-30 fs, FEL photons might induce an emission process $(3 b)$

a resonant enhancement of the $\mathrm{Ne}^{2+}$-to- $\mathrm{Ne}^{+}$ratio for photon energies beyond $41.45 \mathrm{eV}$. The ratio strongly increases by a factor of 2 from 0.037 to 0.075 in the photon energy range from 41.38 to $41.52 \mathrm{eV}$.

In principle, for a resonant excitation, one would expect a much larger effect. However, the typical photon energy bandwidth of FLASH is on the order of about $1 \%$, i.e., 0.4 $\mathrm{eV}$, whereas the typical resonance width is on the order of 5 meV. Thus, due to this bandwidth mismatch, only a small part of the FLASH radiation will excite a resonance with, however, usually a much larger cross section. At a resolution of $22 \mathrm{meV}$, Covington et al. gave a cross section around 55 $\mathrm{Mb}$ for the strong $n=6$ resonance at $41.65 \mathrm{eV}$, compared to $4.45 \mathrm{Mb}$ nonresonant cross section. Thus $\approx 22 / 400=5.5 \%$ of a FLASH pulse will excite the resonance with a 12 times larger cross section. This results in a $\mathrm{Ne}^{2+}$ signal resonantly enhanced by almost $70 \%$. Taking into account the other resonances, this is in excellent agreement with our results of a factor of 2 shown in Fig. 4.

Figure 5 summarizes the possible photoionization pathways that may lead to the resonant or the nonresonant generation of $\mathrm{Ne}^{+}$and $\mathrm{Ne}^{2+}$ states. In all cases, they start with the dominating linear one-photon $2 p$ single ionization $\mathrm{Ne} \rightarrow \mathrm{Ne}^{+}$(1). If followed by a corresponding $\mathrm{Ne}^{+} \rightarrow \mathrm{Ne}^{2+}$ process $(2 a)$, it represents, on one hand, our nonresonant case of $\mathrm{Ne}^{2+}$ generation for photon energies below $41.45 \mathrm{eV}$. If, on the other hand, a resonant $2 s^{2} 2 p^{4}\left({ }^{1} D_{2}\right) n \ell$ state is excited at photon energies above $41.45 \mathrm{eV}(2 b)$, the latter may decay via autoionization to $\mathrm{Ne}^{2+}$ with a lifetime of about $130 \mathrm{fs}$ which results from the corresponding linewidths of $5.1 \mathrm{meV}$ [11]. However, since the lifetime of the excited $2 s^{2} 2 p^{4}\left({ }^{1} D_{2}\right) n \ell$ resonance states is by a factor of $5-10$ longer than the FLASH pulse duration on the order of 20-40 fs around $40 \mathrm{eV}$ photon energy [16], the absorption of a third photon at photon exposures from $10^{16}$ to $10^{17}$ (photons $/ \mathrm{cm}^{2}$ )/ pulse becomes probable as well. Most likely, the excited $n \ell$ electron should be affected, which may be further excited into the continuum leading to $\mathrm{Ne}^{2+}(3 a)$ or de-excited leading to the stimulated emission of a photon and the population of the $\mathrm{Ne}^{+}$ground state $(3 b)$.

The stimulated emission of a photon represents a process of particular interest. With a photon exposure of 3 $\times 10^{16}$ (photons $/ \mathrm{cm}^{2}$ ) / pulse and a resonant cross section of around $50 \mathrm{Mb}$, the resonant excitation or de-excitation probability amounts to already $100 \%$. In the present experiment, due to the bandwidth overlap of about $6 \%$, the resonant absorption probability of a photon, however, is reduced to $10 \%$. For larger photon exposures, above $10^{17}$ (photons $/ \mathrm{cm}^{2}$ )/ pulse, which can be reached by using, e.g., multilayer focusing optics, the absorption probability will increase and also the stimulated emission should be of considerable significance. Such a process has never been observed, so far, in the soft-X-ray regime. Rohringer and Santra discuss stimulated photon emission in a recent theoretical work on $\mathrm{Ne} 1 s \rightarrow 3 p$ excited states [17]. The process would considerably affect the rate equations for the generation and the annihilation of ion species by photoabsorption, which might be experimentally observed by their signal dependence on photon exposure and irradiance beyond the depletion regime.

In view of future experiments, we have further analyzed this behavior by solving the corresponding rate equations for the resonant and the nonresonant case in a simplified model taking into account processes $1,2 a, 2 b$, and $3 b$ from Fig. 5 . For the nonresonant case, the photoionization is described by the cross sections $\sigma_{01}$ and $\sigma_{12}$ for processes 1 and $2 a$, respectively. In terms of the photon exposure $H_{p h}$, which is the photon number per unit area, the corresponding rate equations are given by

$$
\begin{gathered}
\frac{d n_{+}}{d H_{p h}}=\left(1-n_{+}-n_{2+}\right) \sigma_{01}-n_{+} \sigma_{12} \\
\frac{d n_{2+}}{d H_{p h}}=n_{+} \sigma_{12} .
\end{gathered}
$$

$n_{q}\left(H_{p h}\right)$, the photon-exposure-dependent numbers of ion species $q$, is normalized to the initial number of targets, respectively. The solutions of this set of differential equations are

$$
\begin{gathered}
n_{+}=\frac{\sigma_{01}\left(e^{-\sigma_{01} x}-e^{-\sigma_{12} x}\right)}{\sigma_{12}-\sigma_{01}}, \\
n_{2+}=\frac{\sigma_{12}-\sigma_{01}-\sigma_{12} e^{-\sigma_{01} x}+\sigma_{01} e^{-\sigma_{12} x}}{\sigma_{12}-\sigma_{01}},
\end{gathered}
$$

which are depicted in Fig. 6 (red lines) for experimentally obtained cross sections $\sigma_{01}=8.2 \mathrm{Mb}[18]$ and $\sigma_{12}=7 \mathrm{Mb}$ [11].

For the resonant case, the set of differential equations (1) has to be extended to take into account the de-excitation process due to the stimulated emission. In a simple model, this can be done by adding a term $n_{2+} \sigma_{21}$ with $\sigma_{21}$ being the cross section for the stimulated emission process $3 b$, 


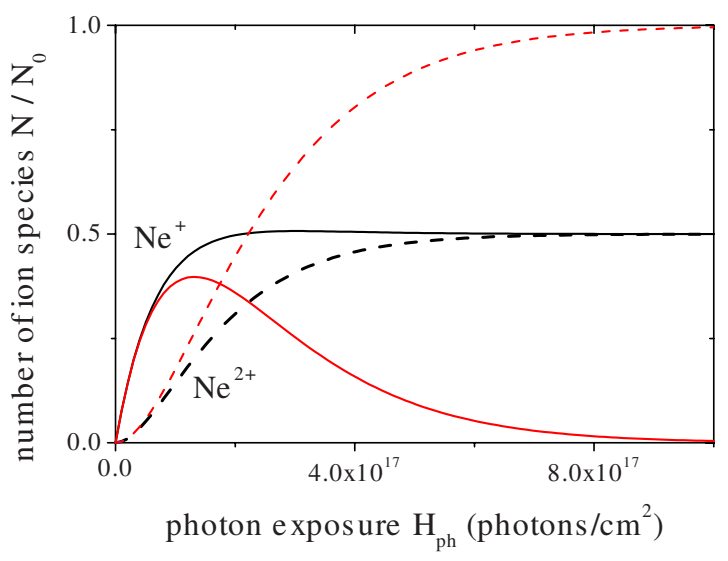

FIG. 6. (Color online) Model curves for the different ion species. The solid line represents $\mathrm{Ne}^{+}$and the dashed lines represent $\mathrm{Ne}^{2+}$. The red lines are the solution for the nonresonant case and the black lines are for the resonant case including the pump cycles. $N_{0}$ is the initial number of neutral neon atoms within the interaction volume.

$$
\begin{gathered}
\frac{d n_{+}}{d H_{p h}}=\left(1-n_{+}-n_{2+}\right) \sigma_{01}-n_{+} \sigma_{12}+n_{2+} \sigma_{21}, \\
\frac{d n_{2+}}{d H_{p h}}=n_{+} \sigma_{12}-n_{2+} \sigma_{21} .
\end{gathered}
$$

In Fig. 6, the solution of this set of differential equations is depicted by the black lines. For comparability, in this model the cross section $\sigma_{21}=\sigma_{12}=7 \mathrm{Mb}$ was chosen as in the nonresonant case.

For high photon exposures, the resonant pump cycle has, in particular, a dramatic effect on the $\mathrm{Ne}^{2+}-$ to- $\mathrm{Ne}^{+}$ratio. In the nonresonant case the $\mathrm{Ne}^{+}$ions will be directly ionized to $\mathrm{Ne}^{2+}$ and the $\mathrm{Ne}^{+}$target will be depleted. Finally, all atoms will be doubly or even higher ionized. In the presence of a long-living resonance state, however, the behavior is different. The excited $\mathrm{Ne}^{+}$ion is de-excited by a further FEL photon back to the ground state by stimulated emission. Therefore, within the FEL pulse duration of a few $10 \mathrm{fs}$, the ground and the excited states are equally populated, which results in the same amount of $\mathrm{Ne}^{+}$and $\mathrm{Ne}^{2+}$ ions. In this simple resonant model the absolute value of the resonant cross section $\sigma_{21}=\sigma_{12}=55 \mathrm{Mb}$ will have the same result at high photon exposures. A more elaborate model including all possible channels as has been done by Markis and Lambropoulos [19] for the nonresonant case should be developed to analyze the stimulated emission process in full detail. Such a model should also include the time structure of the photon pulse. For short pulses, as in the current experiment, when the photon pulse is much shorter than the lifetime of the resonant state, the lifetime has only a minor influence on the $\mathrm{Ne}^{2+}$-to- $\mathrm{Ne}^{+}$ratio. However, in the limit of long pulses due to the decay of the resonant state into $\mathrm{Ne}^{2+}$, finally, all atoms will be doubly ionized. Thus, the ratio of doubly and singly ionized atoms will depend not only on the photon exposure, but also on the time structure of the photon pulse. In future experiments, we will concentrate on this point.

\section{SUMMARY}

We have studied the resonant multiphoton ionization of $\mathrm{Ne}$ atoms in the vicinity of $\mathrm{Ne}^{+}$inner-valence level resonances between 41 and $42 \mathrm{eV}$ photon energies using highly intense free-electron laser radiation and photoion spectroscopy. For all spectra, also the spectral distribution of the FEL pulses was recorded simultaneously by using a special option of the FLASH plane grating monochromator beamline. By correlating the measured $\mathrm{Ne}^{2+}$-to- $\mathrm{Ne}^{+}$ratio with the mean photon energy for each single FLASH pulse, a strong photon energy dependence of the ion ratio was found. The observed enhancement is attributed to resonant two-photon excitation into $\mathrm{Ne}^{2+}$. Taking into account the bandwidth difference between the FEL radiation and the resonance, the observed effect is in excellent agreement with the available crosssection data. For future experiments, we have, in addition, modeled the influence of stimulated photon emission from the excited resonance states on the photoion distribution.

\section{ACKNOWLEDGMENTS}

We are thankful to the FLASH team for delivering the excellent beam to our experiment. This work was supported by the German Ministry for Education and Research (BMBF) within the framework of the priority program FLASH: Matter in the light of ultrashort and extremely intense $\mathrm{x}$-ray pulses and the Deutsche Forschungsgemeinschaft Contracts No. MA2561/4-1, No. RI804/5-1, and No. TI280/ 3-1.
[1] V. Ayvazyan et al., Phys. Rev. Lett. 88, 104802 (2002).

[2] W. Ackermann et al., Nat. Photonics 1, 336 (2007).

[3] H. Mashiko, A. Suda, and K. Midorikawa, Appl. Opt. 45, 573 (2006).

[4] M. Nagasono et al., Phys. Rev. A 75, 051406(R) (2007).

[5] A. A. Sorokin, S. V. Bobashev, T. Feigl, K. Tiedtke, H. Wabnitz, and M. Richter, Phys. Rev. Lett. 99, 213002 (2007).

[6] S. V. Popruzhenko, V. D. Mur, V. S. Popov, and D. Bauer, Phys. Rev. Lett. 101, 193003 (2008).

[7] M. G. Makris, P. Lambropoulos, and A. Mihelič, Phys. Rev.
Lett. 102, 033002 (2009).

[8] L. Hamonou and H. W. van der Hart, J. Phys. B 41, 121001 (2008).

[9] L. Hamonou, H. W. van der Hart, K. M. Dunseath, and M. Terao-Dunseath, J. Phys. B 41, 015603 (2008).

[10] M. Martins, M. Wellhöfer, J. T. Hoeft, W. Wurth, J. Feldhaus, and R. Follath, Rev. Sci. Instrum. 77, 115108 (2006).

[11] A. M. Covington et al., Phys. Rev. A 66, 062710 (2002).

[12] A. A. Sorokin et al., Appl. Phys. Lett. 89, 221114 (2006).

[13] M. Wellhöfer, M. Martins, W. Wurth, A. A. Sorokin, and M. 
Richter, J. Opt. A, Pure Appl. Opt. 9, 749 (2007).

[14] K. Tiedtke et al., J. Appl. Phys. 103, 094511 (2008).

[15] A. A. Sorokin, M. Wellhöfer, S. V. Bobashev, K. Tiedtke, and M. Richter, Phys. Rev. A 75, 051402(R) (2007).

[16] R. Mitzner et al., Opt. Express 16, 19909 (2008).
[17] N. Rohringer and R. Santra, Phys. Rev. A 77, 053404 (2008).

[18] J. M. Bizau and F. J. Wuilleumier, J. Electron Spectrosc. Relat. Phenom. 71, 205 (1995).

[19] M. G. Makris and P. Lambropoulos, Phys. Rev. A 77, 023401 (2008). 